\title{
Update in Management Strategies of Chronic Myeloid Leukemia
}

\author{
Nahla A M Hamed* \\ Hematology Department, Faculty of Medicine, Alexandria University, Egypt
}

Submission: August 17, 2020; Published: September 03, 2020

*Corresponding author: Nahla A M Hamed, Professor of Clinical Hematology, Hematology Department, Faculty of Medicine, Alexandria University, Egypt

\section{Abstract}

The goal of CML treatment in the recent era is to discontinue medication for treatment free remission after achieving a stable deep molecular response. A prerequisite for TKI discontinuation is the assessment of the remaining tumor load by standardized molecular monitoring using high quality quantitative PCR by the international scale. Molecular monitoring post-treatment suspension is also imperative for CML patients attempting TFR.

Keywords:CML: Chronic Myeloid Leukemia; TFR: Treatment Free Remission; DMR: Deep Molecular Response; OS: Overall Survival; ACA Additional Chromosomal Abnormalities; qPCR: Quantitative Polymerase Chain Reaction; ELTS: EUTOS Long Term Survival; CP-CML: Chronic Phase-CML; Mo-MDSCs: Monocytic Myeloid-Derived Suppressor Cells; 2GTKIs: Second Generation Tyrosine Kinase Inhibitors; ELN: European Leukemia Net; NGS: Next Generation Sequencing

\section{Introduction}

Chronic myeloid leukemia (CML) accounts for $15 \%$ of adult leukemias. CML occurs in all age groups with the median age of disease onset is 67 years (SEER statistics) [1]. Prognosis and outcome of CML patients considerably improved and progress in the use of tyrosine kinase inhibitors is ongoing [1]. The concept of treatment free remission (TFR) is considered now part of the management of CML patients. An essential part of the management of CML patients is to assess regularly the remaining tumor load by standardized molecular methods [2].

\section{Update in CML Diagnosis}

\section{Bone marrow}

Bone marrow aspirate and biopsy are recommended at base line for morphologic and cytogenetic evaluation and for quantitative reverse transcriptase (RT-PCR) to establish the presence of quantifiable BCR-ABL1 mRNA transcripts [1]. It provides information for staging in terms of blast and basophil percentages [3]. Bone marrow aspiration is also recommended if a change in therapy is under consideration because of inadequate response or disease progression (drug resistance) to assess for clonal evolution. BCR-ABL1 kinase domain mutation analysis should be done in such circumstances [4].

\section{Cytogenetics}

Cytogenetics should be done in patients with atypical translocation, rare or atypical BCR-ABL1 transcript that cannot be measured by qPCR, treatment failure / resistance to exclude ACA and with progression to AP or BP [4]. High risk ACA include +8 , a second $\mathrm{Ph}$ - chromosome, $\mathrm{i}(17 \mathrm{q}),+19,-7 / 7 \mathrm{q}-, 11 \mathrm{q} 23$, or $3 q 26.2$ aberrations and complex aberrant karyotypes [4]. A FISH test is required If the $\mathrm{Ph}$ - chromosome cannot be identified by cytogenetics but a molecular assay demonstrates BCR-ABL1 [4]. FISH studies may have a false positive range of 1- 5\% depending on the probes used [3].

\section{Quantitative PCR}

The typical BCR-ABL translocation results in e13a2 or e14a2 transcripts [3]. About $1 \%$ of patients may have e $1 \mathrm{a} 2 / \mathrm{a} 3$ transcript. These patients may have a worse prognosis [3]. About 2-4\% of patients harbor atypical BCR-ABL1 transcript lacking ABL1 exon 2 (e13a3 or e14a3) or resulting from atypical BCR breakpoints (e.g. e1a2, e6a2, e8a2, or e19a2) that may yield a false negative PCR by routine probes and (if not tested at diagnosis) would give a false impression that a patient may be in a complete molecular response on TKI [4]. b2a2 cells are more persistent than b3a2 transcripts [4]. 
BCR-ABL1 mutation can be detected by Sanger sequencing (sensitivity: 20\%) and by next generation sequencing (NGS) (sensitivity: 3\%). NGS is the recommended technology to detect BCR-ABL resistance mutation in patients not responding adequately to TKI [4].

\section{Enhanced Detection Methods}

- $\quad$ Digital PCR (dPCR) is more accurate and sensitive than real time qPCR. It detected measurable transcripts that were undetectable by conventional real time qPCR.

- $\quad$ Digital droplet PCR is more predictive of TFR outcomes when tested at the time of TKI discontinuation in CP-CML

- The Light Cycler quantitative reverse transcription (qRT)-PCR system with ABL1 as reference gene (LC/ABL1) and TaqMan systems with the GUSB as reference gene (TM/GUSB). TM/GUSB is a robust and reliable method for monitoring CML patients. It shows improved sensitivity compared with LC/ABL1 [5].

\section{Molecular Response Definitions}

- The term complete molecular response should be avoided and substituted by the term molecularly undetectable leukemia with specification of the number of the control gene transcripts [4].

- Operational cure namely the persistence of detectable leukemic cells in deep molecular response (DMR) while the patients are either on- treatment or off- treatment. This is likely governed by the immune control of minimal residual disease and the type of BCR-ABL1 transcript [4]. The host immune system can protect the patients to develop CML progression derived from the residual leukemic cells existing in these patients [6]. NK cells with increased expression of activating NK receptors were higher in patients who achieved TFR. However, there was no difference in the proportion of CD4+ or CD8+ T cells. T-reg and Mo-MDSCs were collectively decreased in TFR patients, indicating that the effector and suppressor arms of the immune system work together [7]. The immunomodulatory effects of TKIs depend on the type of TKI used.

The proportion of NK cells with cytotoxic potential increased during imatinib treatment. Dasatinib can strongly induce expansion of large granular lymphocytes (T or NK large granular lymphocytes) leading to favorable clinical outcome. No immunological changes were observed during bosutinib therapy. Further, treatment with imatinib, dasatinib or nilotinib can reduce levels of immune suppressive $\mathrm{T}$ cell fraction including Tregs and MDSCs [6].

- The definition of acceptable response to third, fourth or fifth line of CML treatment is not formalized but a BCR-ABL1 transcript level $>1 \%$ or cytogenetic response less than complete $(\mathrm{Ph}+>0 \%)$ are insufficient for optimal survival [4].

\section{Baseline Prognostic Factors}

The three prognostic systems, Sokal, Euro and Eutos were designed to evaluate differences in survival risk at baseline. Most CML patients now die while still in remission from causes other than leukemia. The new EUTOS Long Term Survival (ELTS) risk score was recommended to be used in predicting the rate of death from CML in TKI treated patients. It uses the same simple parameters used in Sokal as hematologic data, spleen size and age. The main difference is the negative prognostic value of age [4]. Resistance to two TKIs, detection of BCR-ABL1 kinase domain mutation or emergence of ACA in $\mathrm{Ph}+\mathrm{ve}$ cells raise progression of disease [4]. High risk ACA predicts a poor response to TKI and a higher risk of progression. Patients with high risk ACA are recommended to be treated as high risk patients [4]. Patients with $\mathrm{ACA} / \mathrm{Ph}+$ at diagnosis should be watched carefully for evidence of therapy failure [1].

\section{Treatment}

\section{Management of chronic phase}

A short course of hydroxyurea may be given in symptomatic patients with high white blood cell or platelet counts while molecular and cytogenetic confirmation of the CML diagnosis is pending [4]. All TKIs are highly effective in newly diagnosed CPCML with long term survival approaching that of age matched controls. The selection of the first line TKI therapy in each patient should be based on the risk score, toxicity profile of TKI, patient's age, ability to tolerate therapy and the presence of comorbid conditions. Those who achieve BCR-ABL1 IS $\leq 10 \%$ at 3 and 6 months and $\leq 1 \%$ at 12 and 15 months after first-line TKI therapy, are considered TKI sensitive patients while those who achieve BCR-ABL1 IS $>10 \%$ at 6 months and $>1 \%$ at 15 months are considered TKI resistant patients [1]. Second generation TKIs (2GTKIs) generally result in faster cytogenetic and molecular responses with less progression to advanced phase CML when compared to imatinib. Yet in randomized clinical trials there are no differences in OS between imatinib and 2GTKIs (dasatinib, nilotinib and bosutinib) [1]. A fifth TKI, radotinib (Supect) has been approved in South Korea only [4].

A first line 2GTKI or a change to a 2 GTKI for faster DMR is not recommended but it may be considered in younger patients with low or intermediate risk disease, patients for whom TFR is a high priority, and women who wish to become pregnant. A change from 2GTKI to imatinib can be considered within 5 years to avoid the risk of serious cumulative toxicity of 2GTKI [4]. Allogeneic HCT is no longer recommended as a first line treatment option for patients with CP-CML [1].

\section{TKI Stopping}

Prerequisites for safe TKI stopping (ELN 2020) (4)

Mandatory 
- $\quad$ CML in first CP only.

- Motivated patient with structural communication.

- Access to high quality quantitative PCR using the international scale (IS) with rapid turn-around of PCR test results.

- $\quad$ Patient's agreement to more frequent monitoring after stopping treatment. This means monthly for the first 6 months, every 2 months for months 6-12 and every 3 months thereafter.

\section{Minimal (stop allowed)}

- $\quad$ First line therapy or second line if intolerance was the only reason for changing TKI.

- $\quad$ Typical e13a2 or e14a2 BCR-ABL transcripts.

- $\quad$ Duration of TKI therapy > 5 years ( $>4$ years for 2GTKI).

- $\quad$ Duration of DMR (MR4 or better) $>2$ years.

- $\quad$ No prior treatment failure.

\section{Optimal (stop recommended for consideration)}

- Duration of TKI therapy $>5$ years.

- $\quad$ Duration of DMR > 3 years it MR4.

- $\quad$ Duration of DMR > 2 years it MR4.5.

Some patients have fluctuating values between MMR and MR4 values which sometimes improve overtime without restarting TKI [4]. The likelihood of the presence of residual BCR-ABL1 in clonal lymphocytes in patients in TFR which may be reducing over time needs to be clarified [5].

More than $80 \%$ of recurrences occur within the first 6-8 months after stopping [4]. Confirmation of loss of MMR on a second occasion is not considered necessary and could delay restarting therapy [4]. Prompt resumption of TKI within 4 weeks of a loss of MMR is recommended with monthly molecular monitoring until MMR is re-established then every 3 months thereafter indefinitely [1]. BCR-ABL1 kinase domain mutation testing should be performed for those who fail to achieve MMR after 3 months of TKI resumption and monthly molecular monitoring should be continued for another 6 months [1].

The RE-STIM study confirmed the safety and success of second TKI discontinuation attempt after a failed first attempt. Major factors significantly associated with TFR outcome were the speed of molecular relapse after the first TKI discontinuation and TKI free duration of $>6$ months after the first attempt of TKI discontinuation [5].

\section{New Drugs and Novel Strategies}

The leukemic stem cells may be quiescent which renders them relatively resistant to TKI therapy. This explains why a substantial proportion of patients relapse when TKI is stopped. Futures TKI independent therapeutic approaches that target the leukemic stem cell will include the addition of pegylated interferon $\alpha 2 b$ to imatinib, dasatinib or nilotinib and combining Bcl-2 inhibitors like venetoclax. Other combinations are the addition of hypomethylating agents, JAK2 inhibitors and immune based therapies including anti-PD1 monoclonal antibodies and dendritic cell vaccines [3].

Asciminib is a potent and specific inhibitor of BCR-ABL1 that binds to the myristoyl pocket of ABL1. It demonstrated clinical activity and good safety and tolerability as monotherapy in heavily pretreated CML patients with resistance or intolerance to at least two prior TKIs in an ongoing phase 1 study (NCT02081378). An ongoing phase 2 clinical trial (NCT03578367) is investigating Asciminib plus imatinib in patients not achieving a DMR with imatinib [5].

\section{Conclusion}

Update in patient management strategies are ongoing TFR. Novel molecular monitoring strategies and new drugs and treatment strategies aimed at improving TFR outcomes are under evaluation and may lead to improvement of TFR prediction and outcomes.

\section{References}

1. NCCN.org (2019) NCCN Clinical Practice Guidelines in Oncology (NCCN Guidelines). Chronic myeloid leukemia. Version 2.2020.

2. Heim D, Ebnother M, Favre G (2019) Chronic myeloid leukemia update 2020. Therapeutische Umschau 76(9): 503-509.

3. Jabbour E, Kantarjianch H (2020) Chronic myeloid leukemia: 2020 update on diagnosis, therapy and monitoring. Am J Hematol 95(6): 691-709.

4. Hochhaus A, Baccarani M, Silver RT, Schiffer C, Apperley JF, et al. (2020) European LeukemiaNet 2020 recommendations for treating chronic myeloid leukemia. Leukemia. 34: 966-984.

5. Annunziata M, Bonifacio M, Breccia M, Castagnetti F, Gozzini G, et al. (2020) Current strategies and future directions to achieve deep molecular response and treatment-free remission in chronic myeloid leukemia. Front Oncol 10: 883.

6. Ureshino H, Shindo T, Kimura S (2020) Role of cancer immunology in chronic myeloid leukemia. Leukemia Research 88: 106273.

7. Slater H (2020) Possible predictors of treatment-free remission in patients with CML. 
This work is licensed under Creative Commons Attribution 4.0 License

DOI:10.19080/CTOIJ.2020.16.555949
Your next submission with Juniper Publishers will reach you the below assets

- Quality Editorial service

- Swift Peer Review

- Reprints availability

- E-prints Service

- Manuscript Podcast for convenient understanding

- Global attainment for your research

- Manuscript accessibility in different formats ( Pdf, E-pub, Full Text, Audio)

- Unceasing customer service

Track the below URL for one-step submission https://juniperpublishers.com/online-submission.php 